\title{
Therapeutic potential of epigallocatechin gallate
}

\author{
Abhinav Anand ${ }^{1}$, Jasneet Chawla ${ }^{2}$, Ankush Mahajan ${ }^{3}$, Neha Sharma', \\ Navneet Khurana ${ }^{1}$
}

\author{
${ }^{1}$ Department of Pharmaceutical Sciences, Lovely Professional University, Punjab, India, ${ }^{2}$ Department of \\ Pharmacology, Dayanand Medical College, Punjab, India, ${ }^{3}$ Department of Phamraceutical Sciences, Khalsa \\ College of Pharmacy, Amritsar, Punjab, India
}

\begin{abstract}
Green tea leaves (Camellia sinensis, Family Theaceae) have been consumed in the form of beverage since long, but their widespread health benefits have been discovered relatively recently. Epigallocatechin gallate (EGCG) is a polyphenol found abundantly in these leaves. It is a potent antioxidant which helps greatly in combating the oxidative stress generated in the body during various physiological processes. Oxidative stress plays a great role in the development of various chronic diseases such as heart diseases, cancer, and neurodegenerative diseases, such as Parkinson's and Alzheimer's diseases. Oxidative stress is also involved in the process of aging. The oxygen radical absorbance capacity assay conducted by the US Department of Agriculture clearly signifies that brewed green tea is a potent antioxidant in comparison to majority of antioxidant rich foods, such as brewed black tea, raw kiwi, lemon juice, and extra virgin olive oil. EGCG has shown promising results in the therapy of various diseases and disorders such as cancers, fatty liver disease, HIV infection, hypertension, Type-II diabetes mellitus, obesity, and neurodegenerative diseases. Some recent studies have revealed novel actions of EGCG, such as interaction with proteins and phospholipids in the plasma membrane. This review presents a comprehensive outlook on the reported pharmacological activities of EGCG along with its green extraction method, pharmacokinetic and toxicity profiles. The data have been collected by several research and review papers obtained from various journal resources including PubMed Central and Elsevier.
\end{abstract}

Key words: Antioxidant, Camellia sinensis, epigallocatechin gallate, oxidative stress

\section{INTRODUCTION}

$\mathrm{E}$ pigallocatechin gallate (EGCG) is a potent antioxidant belonging to the chemical class of polyphenols which is found abundantly in unfermented (green) tea leaves. Green tea has been consumed since long for its relaxing effects, but it has been subjected to tremendous research relatively recently. It has been found out that green tea ensues a plethora of beneficial effects on the overall health due to the presence of EGCG [Figure 1]. ${ }^{[1]}$

Antioxidants are substances which help in scavenging the reactive oxygen species (ROS). These ROS are a number of reactive molecules and free radicals which are chemically the derivatives of molecular oxygen. These ROS are invariably produced during normal aerobic respiration in the cells. These reactive molecules are responsible for a number of adverse events leading to cellular death eventually. ${ }^{[2]}$ There are some intrinsic antioxidant mechanisms such as superoxide dismutase, catalase, and glutathione peroxidases present in the body which neutralize the ROS, and thereby protect the body cells. However, at times, the production of ROS exceeds the capacity of these mechanisms to tackle them. Such a condition is termed as oxidative stress. ${ }^{[3]}$ Oxidative stress is responsible for many adverse events such as lipid peroxidation, physiologic adaptation phenomena, oxidative DNA damage, and signal transduction pathways within the cells. Furthermore, the relation between oxidative stress and several lifestyle-related diseases, aging, chronic and neurodegenerative diseases has been established over time ${ }^{[4]}$ Owing to the fact that the innate antioxidant

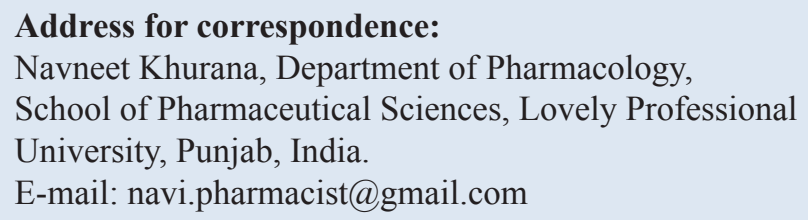

Received: 17-08-2017

Revised: 28-08-2017

Accepted: 02-09-2017 


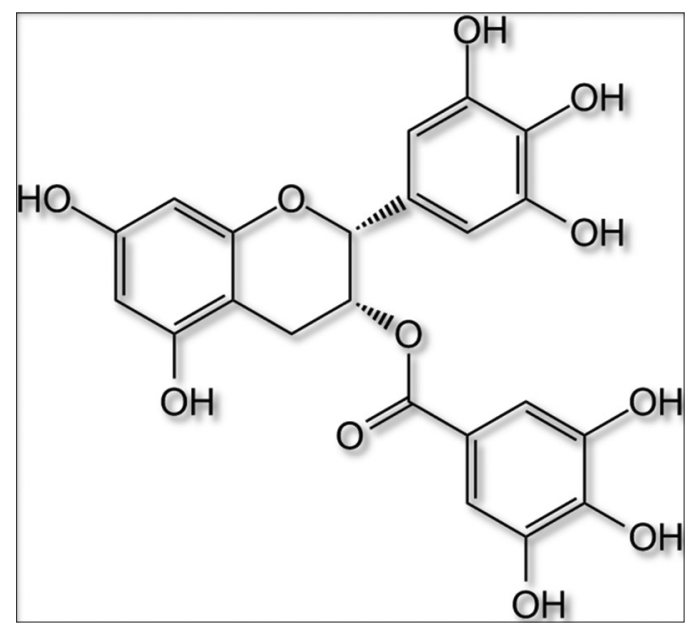

Figure 1: Chemical structure of (-) epigallocatechin gallate

mechanisms of the body are not $100 \%$ sufficient, the need for dietary antioxidant arises. Dietary antioxidants can be defined substances available in the foods that significantly reduce the adverse effects of ROS on normal physiological functions, such as ascorbic acid (Vitamin C) and tocopherol (Vitamin E). As per the oxygen radical absorbance capacity (ORAC) assay conducted by the US Department of Agriculture, EGCG is a potent dietary antioxidant. Its ORAC value exceeds that of various antioxidant rich foods such as raw Kiwi, extra virgin olive oil, lemon juice, brewed black tea. The development of various chronic diseases such as atherosclerosis, cancers, fatty liver disease, heart diseases, and neurodegenerative diseases like Alzheimer's and Parkinson's diseases may be attributed, in part, to the oxidative stress. ${ }^{[5]}$ On the basis of several studies, EGCG has been reported to be of therapeutic value in several diseases. Many companies have been involved in the manufacturing of EGCG tablets. In this review, we shall discuss the therapeutic potential of EGCG, its extraction and toxicity.

\section{EXTRACTION}

Several methods have been proposed for extraction of (-) EGCG from green tea leaves. The widely used process for extraction of polyphenols from green tea leaves is extraction using organic solvents, such as alcohol, and then separating it by adsorption. ${ }^{[6]}$ Green tea polyphenols have also been extracted using microwaves. In this technique, the tea leaves are suspended in organic solvents. The mixture is then irradiated with microwaves ${ }^{[7,8]}$ Another process makes use of a supercritical fluid extraction method along with an absorption system for extraction of green tea polyphenols ${ }^{[9]}$ However, in the abovementioned conventional methods organic solvents such as methanol are extensively used. These solvents ensue varying degrees of toxicity as a complete separation of these solvents from the final is rather difficult. Even slight traces of these solvents are capable of making the polyphenol powder toxic.
However, a novel method was recently proposed which involves a two-step aqueous extraction followed by employment of microfiltration and ultrafiltration, successively [Figure 2].

This method is having a lot of advantages over earlier described methods including no use of any organic solvent, thereby making it a green process, inexpensive nature thereby making it easy to be scaled up and/or down, and bulk extraction of EGCG on industrial scales with acceptable purity. ${ }^{[10]}$

\section{REPORTED PHARMACOLOGICAL ACTIVITIES}

\section{Antineoplastic}

Tea has been consumed as a refreshing beverage since ages, however, its health benefits have been extensively studied recently. It has been proven to have a preventive action in a number of diseases including cancer. Anti-cancer effects of green tea polyphenol, EGCG, have been exhibited by several pre-clinical and clinical studies. The antitumor effects (-) EGCG were discovered from various cancerous cell lines, animal models, and clinical studies. ${ }^{[11-13]}$ Several studies have demonstrated that (-) EGCG plays a pivotal role in prevention of the proliferation of cervical cancer linked with induction of apoptosis and inhibition of telomerase, respectively. ${ }^{[14-16]}$ Several in vitro studies have reported that pure (-) EGCG led to inhibition the growth of oral squamous carcinoma cells and oral epithelial cells. (-) EGCG was also found to halt the growth rate along with arrest of $\mathrm{G}_{2}-\mathrm{M}$ phase in lung cancer cells, both in vitro and in vivo. ${ }^{[17-20]}$

(-) EGCG was also demonstrated to hinder the growth of human hepatocellular carcinoma cells. Downregulation of N-cadherin has also been exhibited by (-) EGCG along with suppression of metastasis of bladder carcinoma cells. ${ }^{[21]}$ Pre-clinical studies have shown that (-) EGCG is capable of inhibiting cell proliferation and inducing cellular death in a wide range of cancer cells including head and neck tumor, prostate cancer, lung cancer, mouse embryonic fibroblast cell tumor, breast cancer, human epidermoid carcinoma, human osteogenic sarcoma, laryngeal squamous carcinoma cells, renal cell carcinoma, nasopharyngeal carcinoma, intestinal tumor, gastric carcinoma, colorectal cancer, hypopharyngeal carcinoma, and pancreatic cancer cells. Various studies have reported that (-) EGCG potently induces apoptosis and arrest of cell cycle in tumor cells but not in their physiological counterparts. ${ }^{[22]}$

\section{In HIV Infection}

EGCG has been reported to have an inhibitory action on replication of HIV-1 before its fusion into the DNA of the 


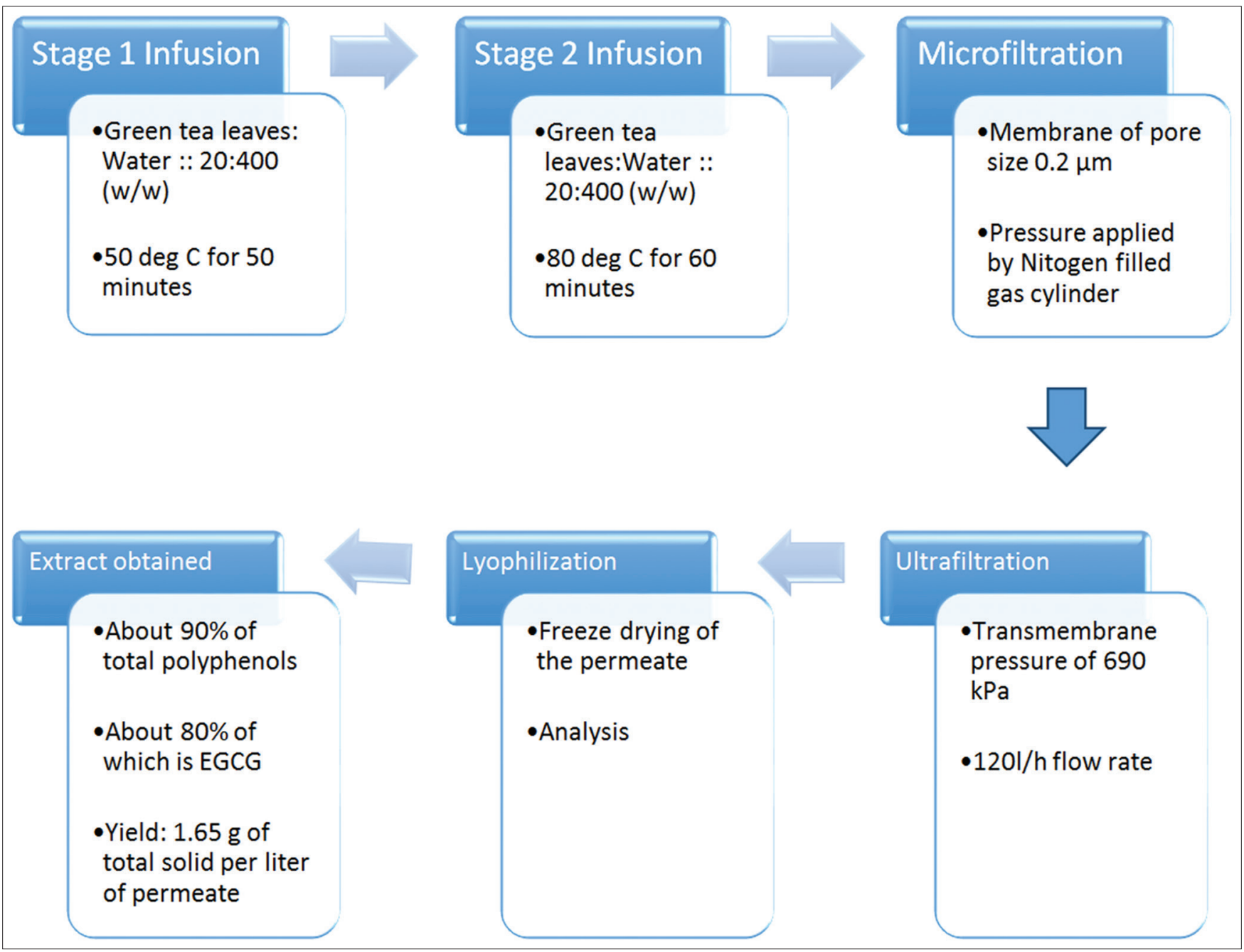

Figure 2: The green method for extraction of epigallocatechin gallate from green tea leaves

host cell. It does so through several proposed mechanisms. However, the specific targets of EGCG are still undefined. The experiments have demonstrated that EGCG was capable to suppress infection caused by HIV-1 and HIV-2, in HIV infected cell lines. Apparently, EGCG acts mainly as an allosteric inhibitor of reverse transcriptase enzyme. It has been proposed that EGCG does so by mechanisms which are different from the currently marketed non-nucleoside reverse transcriptase inhibitors (NNRTIs) that interact directly with the NNRTI binding site on the enzyme. Therefore, EGCG has been viewed as a good candidate for use as an additional anti-HIV agent obtained from natural resources. ${ }^{[23]}$

\section{In Hypertension and Associated Complications}

The plant-derived catechin EGCG leads to endotheliumdependent vasorelaxation by activating endothelial nitric oxide synthase, naturally in endothelial cells by augmenting its protein phosphorylation. ${ }^{[24]}$ A study reported that the acute actions of EGCG in stimulation of generation of nitric oxide from endothelial cells uses PI 3-kinase-dependent pathways. It may partially explain the beneficial effects of EGCG in simultaneously improving cardiovascular and metabolic pathophysiology in spontaneously hypertensive rats. ${ }^{[25]}$ There have been observations made regarding the therapeutic efficacy of EGCG in treatment of hypertension-induced cognitive impairment, probably through its strong antioxidative potential. ${ }^{[26]}$

\section{In Type-II Diabetes Mellitus}

A study has shown that EGCG alters glucose and lipid metabolism in a beneficial manner while significantly enhancing glucose tolerability in diabetic rodents. EGCG has been reported to be involved in downregulation of genes involved in synthesis of fatty acids, triacylglycerol, cholesterol, and gluconeogenesis. Expression of glucokinase mRNA was upregulated in a dose-dependent manner in the liver of $\mathrm{db} / \mathrm{db}$ mice. Dietary supplementation with EGCG can provide a potential contribution to nutritional interventions to prevent and treat type-II diabetes mellitus. ${ }^{[27]}$ 


\section{As Cardioprotective}

Oxidative stress is a major causative factor that forms a link between increased levels of plasma cholesterol with atherogenesis and therefore with myocardial infarction. Hypercholesterolemia leads to production of free radicals in large amounts, thereby increasing mitochondrial respiration and lowering the antioxidant levels. A study reported that EGCG markedly halted the development of abnormalities in tissues, thereby improving the morphology of myocardium. It also revealed that EGCG plays a major role in guarding the cardiovascular system against the diet rich in fats. As per the study, the cardioprotective effect of EGCG was shown by the improvements in the plasma lipid profile, lipid metabolism, antioxidant system, and morphology of myocardial fiber. ${ }^{[28]}$

\section{As Hepatoprotective}

As per a study, histological analyses of liver samples exhibited reduced accumulation of lipids in hepatocytes in EGCG treated mice as compared to mice without EGCG treatment, both groups fed with fat rich diet. It also proved that prolonged treatment with EGCG decreased the development of obesity, symptoms of the metabolic syndrome, and fatty liver. ${ }^{[29]}$ Another study indicated that the reactive species of oxygen and nitrogen generated from mitochondria plays key pathogenetic role in the promotion of liver inflammation and fibrosis. EGCG has been shown to be beneficial in reduction of haptic inflammation and fibrosis. ${ }^{[30]}$

\section{As Nephroprotective}

A group of researchers studied the protective impacts of (-) EGCG in diabetic nephropathy using mice. There was an elevation in levels of plasma glucose, creatinine, urea nitrogen in blood, and protein amounts in urine with decreased overall body weight of the diseased animals. Remarkable reversal of the abovementioned parameters with EGCG treatment was observed. ${ }^{[31]}$ Another study suggested that EGCG exerts a protective effect on rats with obstructive nephropathy by suppression of oxidative stress influenced by free radicals. ${ }^{[32]}$

\section{In Alzheimer's Disease}

Alzheimer's disease is characterized by aggregation of amyloid beta $(A \beta)$ plaques in the brain. ${ }^{[33]}$ These plaques induce a dysfunction in the mitochondria which leads to progression of the disease. ${ }^{[34]}$ EGCG was identified as a mitochondrial restorative compound based on an in vitro study. It was later subjected to in vivo study for the determination of its beneficial effect on mitochondrial function in brain of a double mutant transgenic mouse model (A $\beta$ PP/PS-1) of Alzheimer's disease. EGCG treatment corrected the rates of mitochondrial respiration, matrix metalloproteinases, ROS production, and ATP levels by $50-85 \%$ in mitochondria obtained from the hippocampus, cortex, and striatum. ${ }^{[35]}$ Another study reported that EGCG promoted the non-amyloidogenic pathway dependent of alpha secretase for cleavage of amyloid precursor protein (APP) in $\mathrm{TgAPP}_{\mathrm{sw}}$ transgenic mice overproducing $A \beta .{ }^{[36]}$

\section{In Parkinson's Disease}

Parkinson's disease is a motor neurodegenerative disorder characterized by degeneration of substantia nigra pars compacta which involves oxidative stress, aggregation of $\alpha$-synuclein, dysregulation of redox metal homeostasis, and neurotoxicity. ${ }^{[37]}$ A study reported that EGCG binds to $\alpha$-synuclein by hydrophobic interactions in an unstable manner. It was implied that EGCG could potently remodel the aggregates of $\alpha$-synuclein, thereby acting as a potential disease modifying agent for the treatment of Parkinson's disease and other $\alpha$-synuclein-related disorders. ${ }^{[38]}$

\section{In Osteoporosis}

Osteoporosis is a degenerative disease of bones featuring loss in bone mass and deterioration of microarchitecture of bone tissue which leads to fragile bones. This, in turn increases the susceptibility to fractures, particularly in the wrist, hip, and spine. ${ }^{[39]}$ A study reported EGCG to have a strong stimulatory effect on mesenchymal stem cells derived from human bone marrow progressing toward the osteogenic lineage, as marked by an elevated alkaline phosphatase activity, the upregulation in expression of genes involved in osteogenesis and the formation of bone-like nodules. Further research suggested that EGCG directs osteogenic differentiation through the upregulation of Runt-related transcription factor 2 in a continuous manner.$^{[40]}$ Another study reported that EGCG was able to augment osteogenesis along with other osteoinductive agents through increased expression of bone morphogenetic protein 2. In addition, EGCG was reported to have a positive effect on cell growth, suggesting its safety as a therapeutic agent. ${ }^{[4]]}$

\section{PHARMACOKINETIC AND TOXICITY PROFILE OF EGCG}

\section{Pharmacokinetics}

As per the study, after administration of a single dose of green tea orally ( $20 \mathrm{mg}$ solids/ $\mathrm{kg}$ ) or pure EGCG $(2 \mathrm{mg} / \mathrm{kg})$ to eight human subjects, the pharmacokinetic parameters of EGCG were reported to be as follows Table 1. ${ }^{[42]}$ 


\begin{tabular}{|c|c|}
\hline Parameter & Value \\
\hline $\mathrm{C}_{\text {max }}$ & $77.9 \pm 22.2 \mathrm{ng} / \mathrm{mL}$ \\
\hline AUC & $508.2 \pm 27$ \\
\hline$T_{\text {max }}$ & $1.3-1.6 \mathrm{~h}$ \\
\hline$t_{1 / 2}$ & $3.4 \pm 0.3 \mathrm{~h}$ \\
\hline
\end{tabular}

EGCG: Epigallocatechin gallate

\section{Toxicity}

\section{Acute dermal toxicity}

Topical application was reported to cause a little irritation in guinea pigs and rats but not in rabbits.

\section{Acute oral toxicity}

A single oral dose of $2000 \mathrm{mg} / \mathrm{kg}$ EGCG was reported to be lethal in rats. However, a dose of $200 \mathrm{mg} / \mathrm{kg}$ EGCG ensued no toxicity.

\section{Short-term oral toxicity}

The dietary administration of EGCG to rats for 13 weeks was not shown to be toxic at daily doses up to $500 \mathrm{mg} / \mathrm{kg}$. Similarly, no harmful effects were noted when EGCG was administered in a daily dose of $500 \mathrm{mg} / \mathrm{kg}$ to pre-fed dogs in divided doses. Morbidity was observed on this dose when it was administered as a single-bolus dose to dogs that were fasted before the dosing. However, this model was not considered as a realistic comparison to the human condition. ${ }^{[43]}$

\section{SUMMARY}

EGCG, a polyphenol, is a potent antioxidant obtained from green tea leaves. It has been studied for its beneficial effects in several disorders, diseases, and pathological conditions. It is a safe compound for consumption as it did not show any significant toxicity on normal doses. EGCG has shown promising results in the therapy of various diseases and disorders such as cancers, fatty liver disease, HIV infection, hypertension, type-II diabetes mellitus, obesity, and neurodegenerative diseases (Alzheimer's disease and Parkinson's disease), and osteoporosis. Over time, many researchers have laid out schemes for the extraction of EGCG from green tea leaves, but all those methods involved the use of organic solvents. Recently, a novel method has been proposed which uses only water for the extraction of the compound and is thereby considered as a green method. Some recent studies have revealed novel actions of EGCG, such as interaction with proteins and phospholipids in the plasma membrane. It thereby regulates various cellular processes, such as DNA methylation, signal transduction, mitochondrial function, transcription factors, and autophagy.

\section{REFERENCES}

1. Wolfram S. Effects of green tea and EGCG on cardiovascular and metabolic health. J Am Coll Nutr 2007;26:373S-88.

2. Ray PD, Huang BW, Tsuji Y. Reactive oxygen species (ROS) homeostasis and redox regulation in cellular signaling. Cell Signal 2012;24:981-90.

3. Betteridge DJ. What is oxidative stress? Metabolism 2000;49:3-8.

4. Sharma P, Jha AB, Dubey RS, Pessarakli M. Reactive oxygen species, oxidative damage, and antioxidative defense mechanism in plants under stressful conditions. J Bot 2012;2012:1-26.

5. Haytowitz DB, Bhagwat S. USDA database for the oxygen radical absorbance capacity (ORAC) of selected foods, release 2. Beltsville: United States Department of Agriculture Database; 2010.

6. Anesini C, Ferraro GE, Filip R. Total polyphenol content and antioxidant capacity of commercially available tea Camellia sinensis in Argentina. J Agric Food Chem 2008;56:9225-9.

7. Pan X, Niu G, Liu H. Microwave-assisted extraction of tea polyphenols and tea caffeine from green tea leaves. Chem Eng Process 2003;42:129-33.

8. Quan PT, van Hang T, Ha NH, de Xuan X, Tuyen TN. Microwave-assisted extraction of polyphenols from fresh tea shoot. Sci Technol Dev 2006;9:69-75.

9. Wang CC, Sheu SR, Jang MJ. Analysis of supercritical carbon dioxide extraction for the caffeine and epigallocatechin-3-gallate in green tea. J Biobased Mater Bioenergy 2013;7:208-12.

10. Kumar A, Thakur BK, De S. Selective extraction of (-) epigallocatechin gallate from green tea leaves using twostage infusion coupled with membrane separation. Food Bioprocess Technol 2012;5:2568-77.

11. Conney AH, Lu Y, Lou Y, Xie J, Huang M. Inhibitory effect of green and black tea on tumor growth. Proc Soc Exp Biol Med 1999;220:229-33.

12. Dreosti IE, Wargovich MJ, Yang CS. Inhibition of carcinogenesis by tea: The evidence from experimental studies. Crit Rev Food Sci Nutr 1997;37:761-70.

13. Yang CS, Kim S, Yang GY, Lee MJ, Liao J, Chung JY, et al. Inhibition of carcinogenesis by tea: Bioavailability of tea polyphenols and mechanisms of actions. Proc Soc Exp Biol Med 1999;220:213-7.

14. Noguchi M, Yokoyama M, Watanabe S, Uchiyama M, Nakao Y, Hara K, et al. Inhibitory effect of the tea polyphenol, (-)-epigallocatechin gallate, on growth of cervical adenocarcinoma cell lines. Cancer Lett 2006;234:135-42.

15. Qiao Y, Cao J, Xie L, Shi X. Cell growth inhibition and 
gene expression regulation by (-)-epigallocatechin-3gallate in human cervical cancer cells. Arch Pharm Res 2009;32:1309-15.

16. Yokoyama M, Noguchi M, Nakao Y, Pater A, Iwasaka T. The tea polyphenol, (-)-epigallocatechin gallate effects on growth, apoptosis, and telomerase activity in cervical cell lines. Gynecol Oncol 2004;92:197-204.

17. Okabe S, Suganuma M, Hayashi M, Sueoka E, Komori A, Fujiki H. Mechanisms of growth inhibition of human lung cancer cell line, PC-9, by tea polyphenols. Jpn J Cancer Res 1997;88:639-43.

18. Sadava D, Whitlock E, Kane SE. The green tea polyphenol, epigallocatechin-3-gallate inhibits telomerase and induces apoptosis in drug-resistant lung cancer cells. Biochem Biophys Res Commun 2007;360:233-7.

19. Shim JH, Su ZY, Chae JI, Kim DJ, Zhu F, Ma WY, et al. Epigallocatechin gallate suppresses lung cancer cell growth through Ras-GTPase-activating protein SH3 domain-binding protein 1. Cancer Prev Res (Phila) 2010;3:670-9.

20. Suganuma M, Kurusu M, Suzuki K, Tasaki E, Fujiki H. Green tea polyphenol stimulates cancer preventive effects of celecoxib in human lung cancer cells by upregulation of GADD153 gene. Int J Cancer 2006;119:33-40.

21. Rieger-Christ KM, Hanley R, Lodowsky C, Bernier T, Vemulapalli P, Roth $\mathrm{M}$, et al. The green tea compound, (-)-epigallocatechin-3-gallate downregulates N-cadherin and suppresses migration of bladder carcinoma cells. J Cell Biochem 2007;102:377-88.

22. Chen D, Wan SB, Yang H, Yuan J, Chan TH, Dou QP. EGCG, green tea polyphenols and their synthetic analogs and prodrugs for human cancer prevention and treatment. Adv Clin Chem 2011;53:155-77.

23. Li S, Hattori T, Kodama EN. Epigallocatechin gallate inhibits the HIV reverse transcription step. Antivir Chem Chemother 2011;21:239-43.

24. Lorenz M, Wessler S, Follmann E, Michaelis W, Düsterhöft T, Baumann G, et al. A constituent of green tea, epigallocatechin-3-gallate, activates endothelial nitric oxide synthase by a phosphatidylinositol-3-OHkinase-, cAMP-dependent protein kinase-, and Aktdependent pathway and leads to endothelial-dependent vasorelaxation. J Biol Chem 2004;279:6190-5.

25. Potenza MA, Marasciulo FL, Tarquinio M, Tiravanti E, Colantuono G, Federici A, et al. EGCG, a green tea polyphenol, improves endothelial function and insulin sensitivity, reduces blood pressure, and protects against myocardial I/R injury in SHR. Am J Physiol Endocrinol Metab 2007;292:E1378-87.

26. Wang $\mathrm{MH}$, Chang WJ, Soung HS, Chang KC. (-)-Epigallocatechin-3-gallate decreases the impairment in learning and memory in spontaneous hypertension rats. Behav Pharmacol 2012;23:771-80.

27. Wolfram S, Raederstorff D, Preller M, Wang Y, Teixeira SR, Riegger C, et al. Epigallocatechin gallate supplementation alleviates diabetes in rodents. J Nutr
$2006 ; 136: 2512-8$.

28. Zhong W, Huan XD, Cao Q, Yang J. Cardioprotective effect of epigallocatechin-3-gallate against myocardial infarction in hypercholesterolemic rats. Exp Ther Med 2015;9:405-10.

29. Bose M, Lambert JD, Ju J, Reuhl KR, Shapses SA, Yang CS. The major green tea polyphenol, (-)-epigallocatechin-3-gallate, inhibits obesity, metabolic syndrome, and fatty liver disease in high-fat-fed mice. J Nutr 2008;138:1677-83.

30. Shen K, Feng X, Su R, Xie H, Zhou L, Zheng S. Epigallocatechin 3-gallate ameliorates bile duct ligation induced liver injury in mice by modulation of mitochondrial oxidative stress and inflammation. PLoS One 2015;10:e0126278.

31. Yoon SP, Maeng YH, Hong R, Lee BR, Kim CG, Kim HL, et al. Protective effects of epigallocatechin gallate (EGCG) on streptozotocin-induced diabetic nephropathy in mice. Acta Histochem 2014;116:1210-5.

32. Wu Y, Yu JF, Zhao CG, Sui FX, Teng X, Wu YB. Therapeutic potential of EGCG on acute renal damage in a rat model of obstructive nephropathy. Mol Med Rep 2013;7:1096-102.

33. Editors of encyclopædia britannica. Alzheimer disease. Encycl Br 2016;1-14.

34. Swerdlow RH, Burns JM, Khan SM. The Alzheimer's disease mitochondrial cascade hypothesis: Progress and perspectives. Biochim Biophys Acta 2014;1842:1219-31.

35. Dragicevic N, Smith A, Lin X, Yuan F, Copes N, Delic V, et al. Green tea epigallocatechin-3-gallate (EGCG) and other flavonoids reduce Alzheimer's amyloidinduced mitochondrial dysfunction. J Alzheimers Dis 2011;26:507-21.

36. Rezai-Zadeh K, Shytle D, Sun N, Mori T, Hou H, Jeanniton D, et al. Green tea epigallocatechin-3gallate (EGCG) modulates amyloid precursor protein cleavage and reduces cerebral amyloidosis in Alzheimer transgenic mice. J Neurosci 2005;25:8807-14.

37. Caruana M, Vassallo N. Tea polyphenols in Parkinson's disease. Adv Exp Med Biol 2015;863:117-37.

38. Xu Y, Zhang Y, Quan Z, Wong W, Guo J, Zhang R, et al. Epigallocatechin Gallate (EGCG) inhibits alphasynuclein aggregation: A potential agent for Parkinson's disease. Neurochem Res 2016;41:2788-96.

39. Shen CL, Yeh JK, Cao JJ, Wang JS. Green tea and bone metabolism. Nutr Res 2009;29:437-56.

40. Jin P, Wu H, Xu G, Zheng L, Zhao J. Epigallocatechin3-gallate (EGCG) as a pro-osteogenic agent to enhance osteogenic differentiation of mesenchymal stem cells from human bone marrow: An in vitro study. Cell Tissue Res 2014;356:381-90.

41. Jin P, Li M, Xu G, Zhang K, Zheng LI, Zhao J. Role of (-)-epigallocatechin-3-gallate in the osteogenic differentiation of human bone marrow mesenchymal stem cells: An enhancer or an inducer? Exp Ther Med 2015;10:828-34.

42. Lee MJ, Maliakal P, Chen L, Meng X, Bondoc FY, 
Prabhu S, et al. Pharmacokinetics of tea catechins after ingestion of green tea and (-)-epigallocatechin-3-gallate by humans: Formation of different metabolites and individual variability. Cancer Epidemiol Biomarkers Prev 2002;11:1025-32.

43. Isbrucker RA, Edwards JA, Wolz E, Davidovich A,
Bausch J. Safety studies on epigallocatechin gallate (EGCG) preparations. Part 2: Dermal, acute and short-term toxicity studies. Food Chem Toxicol 2006;44:636-50.

Source of Support: Nil. Conflict of Interest: None declared. 